\title{
Correction: Two pore domain potassium channels in cerebral ischemia: a focus on K2P9.1 (TASK3, KCNK9)
}

Petra Ehling*, Stefan Bittner, Nicole Bobak, Tobias Schwarz, Heinz Wiendl, Thomas Budde, Christoph Kleinschnitz and Sven G Meuth

It has come to our attention that there is an error in the Acknowledgements section of our article [1]. The Acknowledgements section should read:

We are grateful to Ms. Melanie Glaser for excellent technical assistance. We are grateful to Douglas A. Bayliss for providing $\mathrm{K}_{2 \mathrm{P}} 9.1^{-1-}$ animals. This work was supported by the Interdisziplinäres Zentrum für klinische Forschung (IZKF A-54-1) to SGM and HW, the DFG (SFB 581, TP: A10) to SGM and DFG K2P-Forschergruppe 1086 (BU1019/ 9-1 to TB and ME3283/1-1 to SGM) and the Else-KrönerFresenius Foundation (2010_A95 to CK and SGM). PE was a member of the Otto-Creutzfeldt-Center for Cognitive and Behavioral Neuroscience Muenster (OCC).

Received: 1 February 2013 Accepted: 2 February 2013

Published: 4 February 2013

\section{Reference}

1. Ehling P, Bittner S, Bobak N, Schwarz T, Wiendl H, Budde T, Kleinschnitz C,

Meuth SG: Two pore domain potassium channels in cerebral ischemia: a focus on K2P9.1 (TASK3, KCNK9). Exp Trans/ Stroke Med 2010, 2:14.

doi:10.1186/2040-7378-5-3

Cite this article as: Ehling et al:: Correction: Two pore domain potassium

channels in cerebral ischemia: a focus on K2P9.1 (TASK3, KCNK9).

Experimental \& Translational Stroke Medicine 2013 5:3.

*Correspondence: pehling@uni-muenster.de 